\title{
A Black Hole in the Galactic Center Complex IRS 13E?
}

\author{
R. Schödel, A. Eckart, and C. Iserlohe \\ I.Physikalisches Institut, Universität zu Köln, Zülpicher Str.77, 50937 Köln, Germany \\ rainer@ph1.uni-koeln.de, eckart@ph1.uni-koeln.de, iserlohe@ph1.uni-koeln.de \\ and \\ R. Genzel ${ }^{1}$ and T. Ott \\ Max-Planck-Institut für extraterrestrische Physik, Giessenbachstraße, 85748 Garching, \\ Germany \\ genzel@mpe.mpg.de, ott@mpe.mpg.de
}

\begin{abstract}
The IRS 13E complex is an unusual concentration of massive, early-type stars at a projected distance of $\sim 0.13 \mathrm{pc}$ from the Milky Way's central supermassive black hole Sagittarius $A^{*}\left(\operatorname{Sgr} A^{*}\right)$. Because of their similar proper motion and their common nature as massive, young stars it has recently been suggested that IRS 13E may be the remnant of a massive stellar cluster containing an intermediate-mass black hole (IMBH) that binds its members gravitationally in the tidal field of $\operatorname{Sgr} A^{*}$. Here, we present an analysis of the proper motions in the IRS 13E environment that combines the currently best available data with a time line of 10 years $^{1}$. We find that an IMBH in IRS $13 \mathrm{E}$ must have a minimum mass of $\sim 10^{4} \mathrm{M}_{\odot}$ in order to bind the source complex gravitationally. This high mass limit in combination with the absence so far of compelling evidence for a nonthermal radio and X-ray source in IRS 13E make it appear unlikely that an IMBH exists in IRS 13E that is sufficiently massive to bind the system gravitationally.
\end{abstract}

Subject headings: Galaxy: center - Galaxy: nucleus - infrared:stars

\footnotetext{
${ }^{1}$ Also: Department of Physics, University of California, Berkeley, CA 94720

${ }^{1}$ Based on observations at the Very Large Telescope (VLT) of the European Southern Observatory (ESO) on Paranal in Chile
} 


\section{Introduction}

In spite of factors such as a strong tidal field due to the central supermassive black hole Sgr A* (e.g., Schödel et al. 2002; Ghez et al. 2003), or strong stellar winds, which pose serious obstacles for star formation, surprisingly, numerous young (a few times $10^{6}$ yrs), massive stars can be found in the central parsec, e.g., the one to two dozen bright so-called He-stars (with characteristic HeI emission lines, see Krabbe et al. 1995; Paumard et al. 2001). The young, massive stars are mainly concentrated in the region $\sim 10^{\prime \prime}$ in projection around Sgr A* and appear to rotate around Sgr A* in two counter-rotating disks that contain an apparently coeval population of Wolf-Rayet (WR), Luminous Blue Variables (LBV), and O/B-stars (Genzel et al. 2003; Levin \& Beloborodov 2003, also Paumard et al. 2005, in preparation). Also, there are B-type main sequence stars in the immediate vicinity of Sgr A* (e.g., Ghez et al. 2003; Eisenhauer et al. 2005). The presence of the young, massive stars near Sgr A* is not well understood and various explanations for their existence are currently being discussed, such as, e.g., infall and collision of molecular clouds, infall and dissolution of a massive cluster (e.g., see discussions in Genzel et al. 2003), or star formation in a self-gravitating accretion disk (e.g., Nayakshin \& Cuadra 2004; Milosavljevic̀ \& Loeb 2004).

In this context, the IRS $13 \mathrm{E}$ complex is a very intriguing object. Located $\sim 3.5^{\prime \prime}$ in projection from Sgr $A^{*}$, it comprises almost half a dozen massive stars within a projected radius of $\sim 0.25^{\prime \prime}$. These stars appear to be WR stars or O-type supergiants (e.g., Krabbe et al. 1995; Eckart et al. 2004; Maillard et al. 2004). In addition to representing an unusual concentration of bright, early-type stars, the IRS 13E sources also have very similar proper motions (see, e.g., Fig. 18 in Genzel et al. 2003). Maillard et al. (2004) suggested that the IRS 13E complex is the remnant core of a massive star cluster that had fallen into the central parsec and dissolved there. Their main arguments are a) the similar proper motions of the IRS 13E cluster members and b) their finding that the stars in IRS 13E appear to be massive, short-lived, and therefore young. Since one would expect that an association of stars such as IRS 13E should be disrupted by the tidal field of Sgr A*, they speculated that an intermediate-mass black hole (IMBH) in IRS $13 \mathrm{E}$ may prevent its disruption. An IMBH in the core of an infalling cluster would also provide an effective means of inward transportation through increased dynamical friction as has been suggested by Hansen \& Milosavljević (2003) (see, however, Kim, Figer, \& Morris 2004).

The possible existence of an IMBH in the GC is currently a hypothesis of high interest in the field. Therefore we consider it timely to present the newest data on stellar dynamics in IRS 13E. In this letter, we analyze proper motions of the stars in and near the IRS 13E complex, derived from ten years of near-infrared (NIR) speckle and AO observations of the GC stellar cluster. 


\section{Observations and Data Reduction}

Observations of the GC stellar cluster are routinely performed since spring 2002 with the CONICA/NAOS NIR camera/adaptive optics (AO) system at the ESO VLT unit telescope 4 on Cerro Paranal in Chile. For this work, we used K-band (and some H-band) imaging data. After standard data reduction (sky subtraction, dead pixel correction, flat fielding, shiftand-add) the final images were Lucy-Richardson (LR) deconvolved and beam restored with a Gaussian beam of $\sim 60$ mas (40 mas for H-band images, respectively), corresponding to the diffraction limit of the VLT at $2.2 \mu \mathrm{m}$. Stellar positions were extracted with StarFinder (Diolaiti et al. 2000). They were transformed into a coordinate system relative to Sgr A* with the aid of 18 reference stars with well known positions and proper motions, taken from (Ott 2004). Each of the data sets was divided into two parts that were reduced and analyzed independently. Uncertainties of the source positions were thus obtained by a comparison of two independent measurements for each epoch.

We added to these data Gemini North AO H and K imaging data from July 2000. The images are part of the Gemini North Galactic Center Demonstration Science Data Set. Both images were LR deconvolved and beam restored. Source positions were extracted in the way described above (using the two independent measurements provided by the $\mathrm{H}$ and $\mathrm{K}$ images). In order to obtain a larger time baseline, we also used SHARP speckle imaging data from July 1995, June 1996, and June 2001. Details on the observation and reduction of SHARP imaging data may be found, e.g., in Eckart \& Genzel (1997). Again, we applied LR deconvolution and used two independent data sets for each epoch.

\section{Dynamics in the IRS 13E complex}

The IRS 13E sources are named in Fig. 1. Eckart et al. (2004) label E3 and E4 as E3c and E3N, respectively. Maillard et al. (2004) noted that E3 is a multiple source and named the two components E3A and E3B. On the image presented here, E3 appears to have more than two components. We just label the brightest one of them E3 (the image in Fig. 1 is based on data with a Strehl $\geq 40 \%$ in contrast to the Gemini image of Strehl $~ 5 \%$ used by Maillard et al. (2004)). As concerns E5, it appears highly confused with other sources and/or extended.

Proper motions of the stars were determined by linear fits to the measured timedependent positions with their respective uncertainties (adopting a GC distance of $7.9 \mathrm{kpc}$ from Eisenhauer et al. 2003). In Fig. 1, the derived proper motions of all stars in and near IRS $13 \mathrm{E}$ are shown superposed on an image. The common proper motion of the IRS 13E 
cluster members stands out clearly. We identify five stars within a region of about $0.5^{\prime \prime}$ diameter that could be part of IRS 13E (see also Table 1). There may be further potential members, but they are either too weak, embedded in extended emission, or too close to brighter stars, such that no reliable proper motions could be determined. The proper motion of E6, while pointing into the same direction as the ones of the other IRS 13E sources, is significantly smaller in magnitude. Also, Maillard et al. (2004) note that E6 is extincted much less than the other IRS 13E stars. It is therefore questionable whether E6 forms part of IRS 13E and we omit it from the following analysis. The proper motion of E5 could not be reliably determined.

Maillard et al. (2004) considered that IRS 13E might be bound by an IMBH. From spectroscopically estimated radial velocities for E2 and E4 and assuming that the hypothetical IMBH is located halfway between the two stars, they estimated its mass to $\sim 1000 \mathrm{M}_{\odot}$. With the proper motions, we can derive the minimum mass that is required to bind the IRS 13E cluster members. Here, it is first necessary to subtract the systemic motion of IRS 13E. Subsequently, the residual velocities of the individual sources can be used for estimating the mass in IRS 13E under the assumption that it is a gravitationally bound system. Since we cannot know the true systemic velocity, we examined three cases: a) Using the average proper motion of the ensemble, b) using the proper motion of E1, and c) using the proper motion of E4. Thus, all realistic cases, even extreme ones, should be covered. In the following, we limit our analysis to case a), which is the most conservative one because it results in the lowest enclosed masses.

The average proper motion of IRS 13E, derived from the mean of the velocities of sources E1, E2, E3, and E4, is $248 \pm 25 \mathrm{~km} \mathrm{~s}^{-1}$ westwards and $80 \pm 48 \mathrm{~km} \mathrm{~s}^{-1}$ northwards. Here, we chose an unweighted average because we cannot know which one of the individual proper motions corresponds best to the possible systemic velocity. The proper motions of the IRS 13E sources after subtraction of a systemic velocity corresponding to their mean motion are shown in the left panel of Fig. 2.

A first rough estimate of the mass needed to bind IRS $13 \mathrm{E}$ can be obtained from the residual velocities, through $M=\left\langle v^{2}\right\rangle R / G$, where $G$ is the gravitational constant and $R \approx$ $10 \mathrm{mpc}$ the size of the system. With $\left\langle v_{R A}^{2}\right\rangle \approx 50 \mathrm{~km} \mathrm{~s}^{-1}\left(\left\langle v_{D e c}^{2}\right\rangle \approx 100 \mathrm{~km} \mathrm{~s}^{-1}\right)$ we obtain $M \approx 5600 \mathrm{M}_{\odot}\left(M \approx 22500 \mathrm{M}_{\odot}\right)$.

We chose two more refined approaches to estimating the mass of a putative IMBH that gravitationally binds IRS 13E: a) Estimating the mass in IRS 13E with the Leonard-Merritt (LM) mass estimator (eq. 19 in Leonard \& Merritt 1989) and b) deriving a lower limit on the mass through the relation $\frac{R v_{\text {proj }}^{2}}{2 G} \leq \frac{r v_{\text {esc }}^{2}}{2 G}=M$, with $R$ being the projected distance of a star from the IMBH, $v_{\text {proj }}$ its velocity projected onto the plane of the sky, $r$ its real distance 
from the IMBH, $v_{\text {esc }}$ its escape velocity, $G$ the gravitational constant, and $M$ the mass of the IMBH.

Taking into account the unknown location of the black hole, we examined a field of $\sim \pm 0.5^{\prime \prime}$ in right ascension and declination around the center of IRS $13 \mathrm{E}$ with a grid step of $0.01^{\prime \prime}$. The residual proper motion of each star results in a required lower mass for the IMBH - dependent on its location - in order to bind the star to IRS 13E. Thus, one can obtain four maps for the sources E1, E2, E3, and E4. The four maps were combined by taking the maximum (otherwise the system would not be bound) of the calculated mass limits at a given location. We show the resulting map of lower mass limits in the right panel of Fig. 2. The significance of the calculated masses over the field is $\geq 4 \sigma$. Since E1 has the proper motion with the strongest deviation from the mean proper motion of the ensemble, it is the star that gives the strongest constraints. There is a narrow minimum in the mass map for black hole locations close to E1 $\left(\sim 0.1^{\prime \prime} \mathrm{NW}\right.$ of E1). In this most conservative case, the mass of the black hole would have to be larger than $7000 \mathrm{M}_{\odot}$ (with a significance of $4 \sigma$ ). If the black hole were located between E2 and E4, the location used by Maillard et al. (2004) for their mass estimate, it would have to have a mass $>15000 \mathrm{M}_{\odot}($ significance $>10 \sigma)$.

These are only lower limits that rely on projected velocities and conservative estimates. If IRS $13 \mathrm{E}$ is indeed a bound system, a more realistic mass might be derived with the LM estimator. Again, there is a minimum near E1 for the black hole mass. However, even in this conservative case, the LM estimator gives a mass of $\sim 50000 \pm 15000 \mathrm{M}_{\odot}$.

If we include the source E6 in the above described analysis, the required black hole masses would at least double, due to the increased velocity gradient across the complex. Hence, we conclude that it is safe to assume that the required mass to bind the members of the IRS $13 \mathrm{E}$ complex must be at least $10^{4} \mathrm{M}_{\odot}$. Assuming the extreme case that the 4 brightest stars in IRS $13 \mathrm{E}$ are similarly massive as the binary IRS $16 \mathrm{SW}\left(\geq 100 \mathrm{M}_{\odot}\right.$, see Ott et al. 1999), it appears conservative to assume that the stellar mass in IRS 13E does not exceed $10^{3} \mathrm{M}_{\odot}$ and can thus be neglected in this analysis.

\section{Discussion}

IRS $13 \mathrm{E}$ is located at a projected distance of $\sim 3.5^{\prime \prime}$ or $\sim 130 \mathrm{mpc}$ from the $3.5 \times$ $10^{6} \mathrm{M}_{\odot}$ black hole Sgr $\mathrm{A}^{*}$. It may be associated with the counter-rotating disk of young stars (Genzel et al. 2003). In this case, it should be located at $\sim 120 \mathrm{mpc}$ behind the plane of the sky. With a radius of $\sim 0.25^{\prime \prime}$, a mass of roughly $1000 \mathrm{M}_{\odot}$ then would be sufficient to protect the system from tidal disruption. However, as the analysis above shows, the real 
constraints for binding IRS 13E gravitationally result from the intrinsic proper motions of the sources in this complex. We took into account the unknown location of the hypothetical black hole and various possibilities for the systemic motion of IRS 13E in the gravitational potential of Sgr $\mathrm{A}^{*}$. In the most conservative case, the minimum mass to bind IRS 13E was found to be $7000 \pm 1800 \mathrm{M}_{\odot}$ (with a significance of $4 \sigma$ ). This would confine the IMBH to a narrow region $\sim 0.1^{\prime \prime} \mathrm{NW}$ of $\mathrm{E} 1$. Outside this region the required mass is greater than $10^{4} \mathrm{M}_{\odot}$. The LM mass estimator results in even higher masses.

It is generally accepted that accreting black holes are associated with non-thermal radio and X-ray emission. One may argue that an IMBH in IRS 13E could be "starved", similar to Sgr A* (see, e.g., Melia \& Falcke 2001), with its emission below the detection limit of current telescopes. However, there are several pieces of evidence that the IRS 13E cluster is closely associated with the gas and dust of the mini-spiral: First, there is the close spatial relation between IRS 13E and emission from warm dust (see, e.g., the high-resolution AO $3.8 \mu \mathrm{m}$ images in Eckart et al. 2004). Paumard et al. (2004) present an analysis of the mini-spiral that also supports a close relation between IRS 13E and the ISM. Also, the stars in IRS 13E, with some of them being WR stars, may present favorable sources of gas and dust. Moultaka et al. (2004) and Moultaka et al. (2005) present spectroscopic evidence for absorption/emission due to gas and dust intrinsic to IRS 13E and for interaction between winds from IRS 13E and the surrounding ISM. Hence, the situation for a hypothetical black hole in IRS 13E appears to be different from Sgr A*, with plenty of material available for accretion.

As for mm/radio emission, Eckart et al. (2004) show that the $13 \mathrm{~mm}$ point source observed by Zhao \& Goss (1998) in the IRS 13E cluster is most likely due to thermal emission from the $4 \mu \mathrm{m}$-excess source E3c in the center of IRS 13E, a dusty WR star (see also Moultaka et al. 2005). The two components E3A and E3B are also proposed as dusty WR stars in the analysis by Maillard et al. (2004). As for X-ray emission, Baganoff et al. (2003) report an X-ray source that they associate with IRS 13E. Using the position of this source and of Sgr $\mathrm{A}^{*}$ as listed in their Table 1, we located this X-ray source in the NIR reference frame. Its position is marked with a $1 \sigma$ error box in Fig. 1 . The X-ray source appears offset from IRS 13E as also discussed by Maillard et al. (2004) . Coker et al. (2002) interpret the spectrum of the source as consistent with that of a post-LBV WR colliding wind binary (although none of the bright IRS 13E members appears to be associated with the source, see Fig. 1). As for a very recent analysis of this X-ray source, it appears to be located a few $0.1^{\prime \prime}$ further north than indicated in Fig. 1, so that E5 lies within the positional uncertainty. No variability of the source was found and it appears to be explained well by colliding winds (F. Baganoff, priv. comm.). Therefore there appears to be no compelling evidence for an accreting IMBH in IRS 13E. Furthermore, spectroscopy at $4 \mu \mathrm{m}$ (Moultaka et al. 2004) and 
$5 \mu \mathrm{m}$ (Moultaka et al. 2005) does not reveal any strong broad lines or emission from highly excited ions as they might be expected near an accreting black hole.

Infall and dissolution of a massive cluster into the central parsec seems an attractive hypothesis for delivering young stars to the region near Sgr A*. Generally, the conditions required for a cluster to form near the GC and to fall in towards the central parsec to deposit young, massive stars there within their lifetime, are extreme, i.e., high core densities and large cluster masses (see discussions in Gerhard 2001; Genzel et al. 2003; Kim \& Morris 2003; Kim, Figer, \& Morris 2004; Maillard et al. 2004). An IMBH in the core of such a cluster was suggested by Hansen \& Milosavljević (2003) as a means of increasing the efficiency of dynamical friction on the cluster, and thus to relax somewhat the demands on its total mass and core density. Kim, Figer, \& Morris (2004), however, conclude that an IMBH in the cluster core must comprise an unrealistic $\sim 10 \%$ of the total cluster mass in order for this mechanism to be effective.

From the proper motions presented here, the required minimum mass for a hypothetical IMBH in order to bind the members of IRS $13 \mathrm{E}$ was derived to be $\gtrsim 10^{4} \mathrm{M}_{\odot}$. Portegies-Zwart \& McMillan (2002) analyze the growth of an IMBH by runaway collisions in a dense stellar cluster and conclude that such an object may form in dense clusters and contain of the order $0.1 \%$ of the total stellar mass. Given the high mass estimate for an IMBH in IRS 13E derived in this work, this would mean an unrealistically high mass of $>10^{6} \mathrm{M}_{\odot}$ for the progenitor cluster. In this context it is also important to point out the results of Reid \& Brunthaler (2004) that exclude the existence of a secondary black hole with masses $\gtrsim 10^{4} \mathrm{M}_{\odot}$ and semimajor axes between $10^{3}$ and $10^{5} \mathrm{AU}$ (corresponding to $\sim 0.1^{\prime \prime}$ to $12.5^{\prime \prime}$ angular distance from Sgr $\left.A^{*}\right)$. We cannot yet exclude an IMBH of up to a few thousand solar masses in IRS 13E, but our results suggest that in this case IRS 13E must be in the process of dissolution. In this context, we would like to point out the recent work by Levin, Wu and Thommes (astroph/0502143). They have simulated the infall and dissolution of a stellar cluster with an IMBH in the GC and concluded that a hypothetical IMBH that binds the IRS 13E complex could not have delivered all of the young stars in the GC to their present location.

The positions and proper motions of the IRS 13E stars are consistent with their being part of the counter-clockwise disk/ring of young stars (Genzel et al. 2003, see also T. Paumard et al., 2005, in preparation). As for the unusual clustering of the stars in IRS 13E, the complex may be, on the one hand, a (quasi-)permanent feature, i.e. gravitationally bound by a hypothetical IMBH. Our analysis shows that in this case, if the star E1 is indeed part of the complex, the IMBH would have to be unsually massive. This makes its existence questionable because of the required large mass of the progenitor cluster and because of the lack of a clear identification of a non-thermal, variable X-ray source centered within IRS 13E. 
However, we cannot completely discard the IMBH hypothesis. On the other hand, IRS 13E may be a temporary feature, i.e., either a chance association (which is appears somewhat unlikely, even if the stars are part of a disk/ring) or a cluster in the process of dissolution. At this point, we would like to point out that an unusual concentration of co-moving stars has recently also been identified among the members of the IRS 16 complex in the clockwise rotating disk of stars (see Lu 2004). Detailed future studies of the proper motions, line-ofsight velocities, distribution, and spectral types of the stars in the central parsec of the GC nuclear cluster are clearly needed in order to understand better the phase-space clustering of the stars in the IRS $13 \mathrm{E}$ and IRS 16 complexes.

Based on observations obtained at the Gemini Observatory, which is operated by the Association of Universities for Research in Astronomy, Inc., under a cooperative agreement with the NSF on behalf of the Gemini partnership: the National Science Foundation (United States), the Particle Physics and Astronomy Research Council (United Kingdom), the National Research Council (Canada), CONICYT (Chile), the Australian Research Council (Australia), CNPq (Brazil) and CONICET (Argentina).

\section{REFERENCES}

Baganoff, F. K., Maeda, Y., Morris, M., et al. 2003, ApJ, 591, 891

Coker, R. F. and Pittard, J. M. and Kastner, J. H. 2002, A\&A, 383, 568

Diolaiti, E., Bendinelli, O., Bonaccini, D., et al. 2000, A\&AS, 147, 335

Eckart, A. \& Genzel, R.1997, MNRAS, 284, 576

Eckart, A., Genzel, R., Ott, T., \& Schödel, R. 2002, MNRAS, 331, 917

Eckart, A., Moultaka, J., Viehmann, T., Straubmeier, C., \& Mouawad, N. 2004, ApJ, 602, 760

Eisenhauer, F., Schödel, R., Genzel, R., et al. 2003, ApJ, 597, L121

Eisenhauer, F., Genzel, R., et al. 2005, ApJ, in press, astro-ph/0502129

Genzel, R., Schödel, R., Ott, T., et al. 2003, ApJ, 594, 812

Gerhard, O. 2001, ApJ, 546, L39

Ghez, A. M., Duchêne, G., Matthews, K., et al. 2003, ApJ, 586, L127 
Hansen, B. M. S. \& Milosavljević, M. 2003, ApJ, 593, L77

Kim, S. S., \& Morris, M. 2003, ApJ, 597, 312

Kim, S. S. and Figer, D. F. and Morris, M. 2004, ApJ, 607, L123

Krabbe, A., Genzel, R., Eckart, A., et al. 1995, ApJ, 447, L95

Leonard, P. J. T. and Merritt, D. 1989, ApJ, 339, 195

Levin, Y. and Beloborodov, A. M. 2003, ApJ, 590, L33

Lu, J., Ghez, A., Morris, M. \& et al. 2004, AAS, 205, 8506.

Ott, T. and Eckart, A. and Genzel, R. 1999, ApJ, 523, 248

Maillard, J. P., Paumard, T., Stolovy, S. R., \& Rigaut, F. 2004, A\&A, 423, 155

Melia, F. \& Falcke, H. 2001, ARA\&A, 39, 309

Milosavljevic̀, A., \& Loeb, A. 2004, ApJ, 604, L45

Moultaka, J., Eckart, A., Viehmann, T., Mouawad, N., Straubmeier, C., Ott, T., \& Schödel, R. 2004, A\&A, 425, 529

Moultaka, J., Eckart, A., Viehmann, T., \& Schödel, R. 2005, submitted to A\&A

Nayakshin, S. \& Cuadra, J. 2005, submitted to A\&A, astro-ph/0409541

Ott, T. 2004, Ph.D. Thesis, Ludwig-Maximilians-Universität, München

Paumard, T., Maillard, J. P., Morris, M., \& Rigaut, F. 2001, A\&A, 366, 466

Paumard, T., Maillard, J. P., \& Morris, M. 2004, A\&A, 426, 81

Portegies Zwart, S. F. and McMillan, S. L. W. 2002, ApJ, 576, 899

Portegies Zwart, S. F. and McMillan, S. L. W. and Gerhard, O. 2003, ApJ, 593, 352

Reid, M. J. and Brunthaler, A. 2004, ApJ, 616, 872

Schödel, R., Ott, T., Genzel, R., et al. 2002, Nature, 419, 694

Zhao, J. \& Goss, W. M. 1998, ApJ, 499, L163 


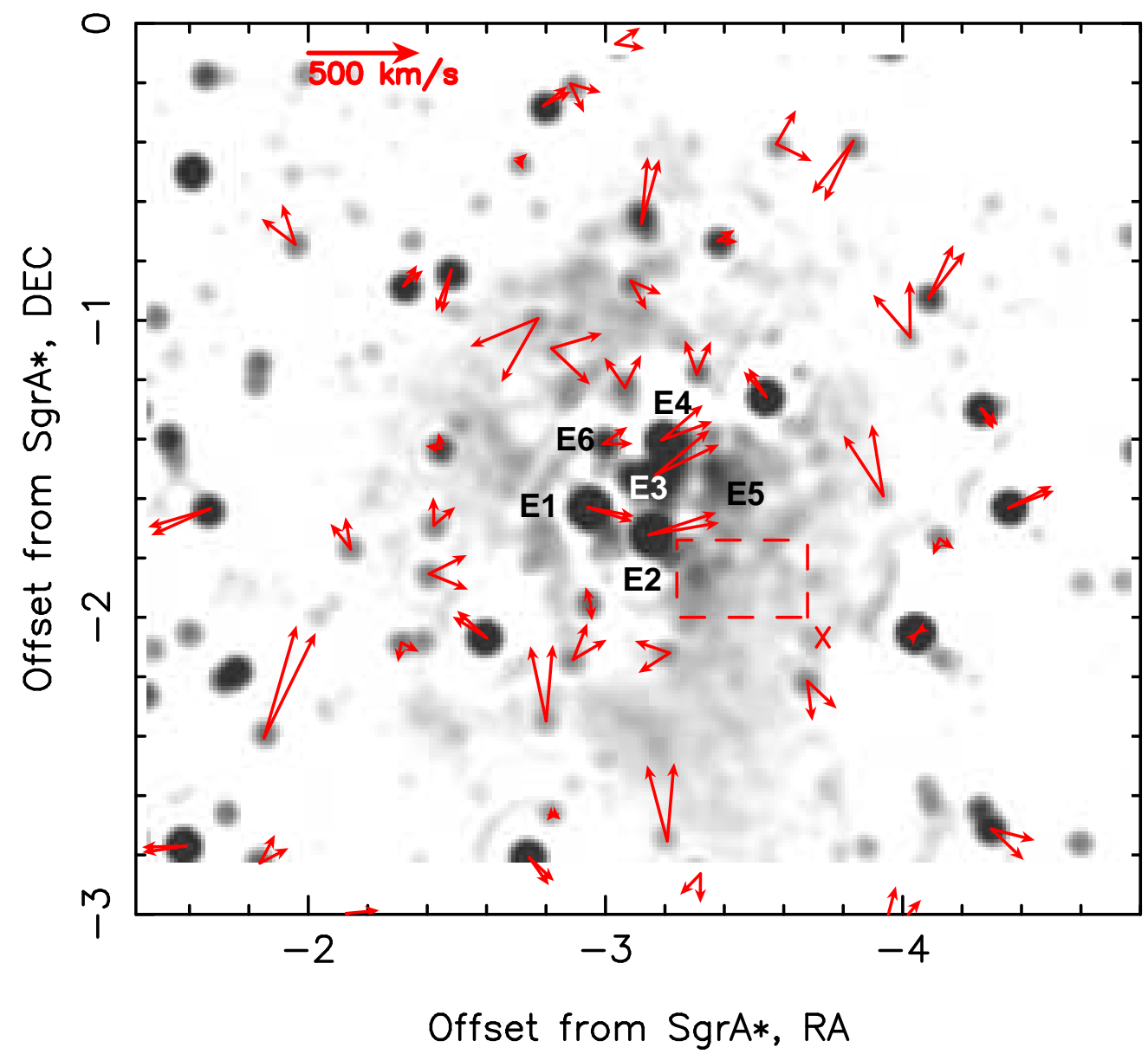

Fig. 1.- Proper motions of stars in the vicinity of IRS 13E. The underlying image is a LRdeconvolved, beam-restored (60 mas FWHM Gaussian beam) NACO K-band image from 8 July 2004. Two arrows are shown for each source, indicating the $\pm 3 \sigma$ uncertainty of the direction of its proper motion. The lengths of the arrows correspond to the magnitude of the respective velocity. The dashed box marked with an "X" designates the position ( $1 \sigma$ uncertainty) of the X-ray source near IRS 13E reported by Baganoff et al. (2003). 

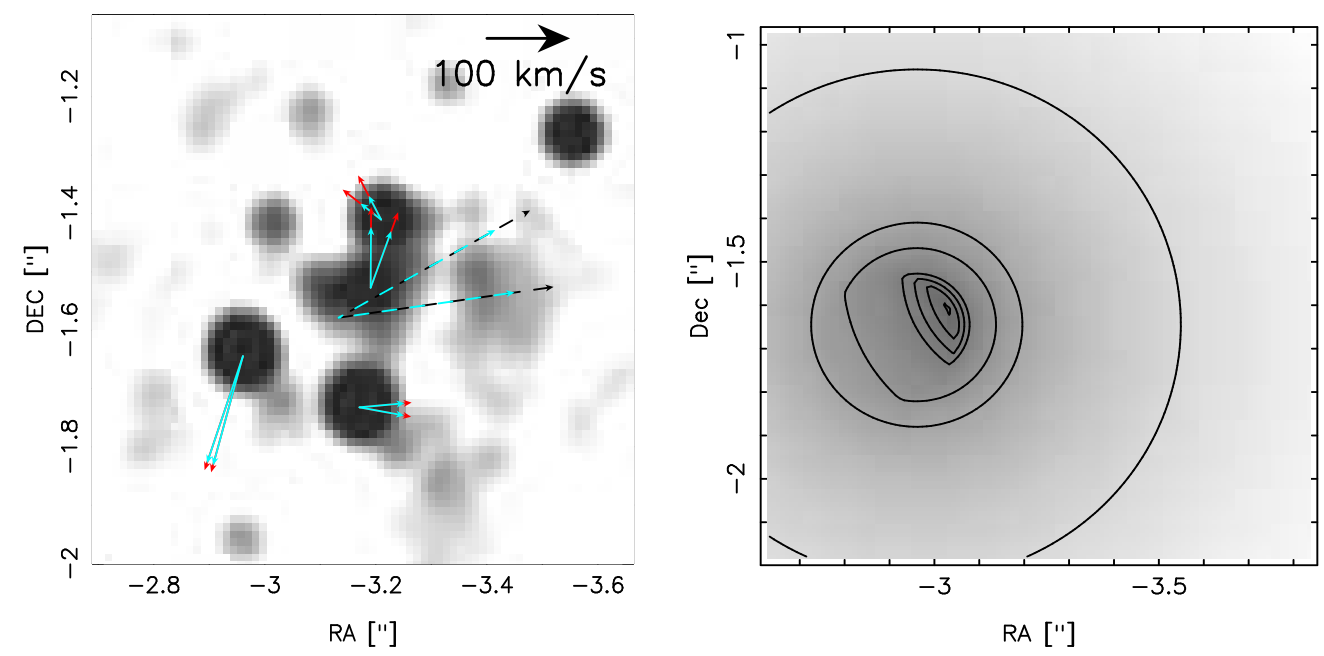

Fig. 2.- Left panel: Zoom into the image shown in Fig. 1. Shown are the residual proper motions of the IRS 13E sources after subtraction of the average projected velocity (indicated by the dashed arrows) of the sources E1, E2, E3, and E4. The opening angles define the $1 \sigma$ uncertainty of the directions. The superposed light grey arrows give the magnitude of the velocities after subtraction of a $1 \sigma$ error. Right panel: Minimum mass required for an IMBH in order to gravitationally bind IRS 13E. This mass depends on the location of the IMBH and was calculated with the residual velocities of the IRS $13 \mathrm{E}$ sources. Contours at 7, 8, 9, $10,15,20$, and $50 \times 10^{3} \mathrm{M}_{\odot}$, growing larger toward lighter shades of gray. The significance of the masses is $4-16 \sigma$. 
Table 1: Positions and velocities of sources in IRS 13E (labeling see Fig. 1).Positions are given as offsets from $\mathrm{Sgr} \mathrm{A}^{*}$ in arc-seconds at the epoch 2004.73.

\begin{tabular}{ccccc} 
Source ID & RA ["] & Dec ["] & $\mathrm{v}_{R A}\left[\mathrm{~km} \mathrm{~s}^{-1}\right]$ & $\mathrm{v}_{\text {Dec }}\left[\mathrm{km} \mathrm{s}^{-1}\right]$ \\
\hline \hline E1 & -2.961 & -1.645 & $-206 \pm 4$ & $-51 \pm 4$ \\
E2 & -3.171 & -1.732 & $-311 \pm 4$ & $77 \pm 8$ \\
E3 & -3.191 & -1.527 & $-265 \pm 17$ & $171 \pm 11$ \\
E4 & -3.210 & -1.411 & $-210 \pm 9$ & $124 \pm 16$ \\
E6 & -3.013 & -1.422 & $-128 \pm 16$ & $41 \pm 13$ \\
\hline
\end{tabular}

